\title{
Numerical and Experimental Investigation of the Heat-Affected Zone in a Laser-Assisted Machining of Ti-6Al-4V Alloy Process
}

\author{
Nancy Yang ${ }^{\mathrm{a}}$, Milan Brandt and Shoujin Sun \\ CAST Cooperative Research Centre, IRIS, Swinburne University of Technology, \\ PO Box 218 Mail H66, Hawthorn VIC 3122, Australia \\ ajyang@swin.edu.au
}

\begin{abstract}
Keywords: Finite element modelling (FEM), Heat-affected zone (HAZ), Laser-assisted machining (LAM), Ti6Al4V alloy
\end{abstract}

\begin{abstract}
The aim of this work is to develop a 3-D transient finite element model for a moving Gaussian laser heat source to predict the depth of the heat-affected zone (HAZ) and temperature distribution in a Ti6Al4V alloy workpiece. It is found that the temperature profile and depth of HAZ are strongly dependent on the parameters of the laser beam. The thermal model simulations are compared with results produced by experimental work and these show close agreement.
\end{abstract}

\section{Introduction}

Titanium alloys have been widely used in the aerospace, biomedical and automotive industries because of their high strength-to-weight ratio and superior corrosion resistance. However, it is very difficult to machine these alloys due to their poor machinability [1].

Laser assisted machining (LAM) is a new and innovative manufacturing process that has been investigated as an alternative to conventional machining of hard and/or difficult-to-process materials such as titanium alloys [2-7]. This heat-assisted process induces a detrimental heataffected zone (HAZ) in the part. Knowledge of the effect of the laser without machining makes it possible to find the optimum condition for LAM. For instance, the entire HAZ can be removed by machining [8].

A 3-D transient finite element model for a moving Gaussian laser heat source was developed to predict the temperatures generated in the workpiece and validated through actual temperature measurements. The computed temperature distribution correlated with the HAZ via experimental tests and a critical temperature range corresponding to the formation of the HAZ was then identified.

\section{Thermal Modelling}

In this work, the estimation of heat treatment is based on the following assumptions:

- The laser beam is regarded as a base mode Gaussian beam vertically directed at the workpiece.

- Both the laser beam and coordinate system are fixed while the workpiece move in the - $y$ direction with a constant velocity $U$.

- The thermo-physical properties are dependent on temperature.

- $\quad$ The treated material is homogeneous.

- The ambient temperature is $295 \mathrm{~K}$.

- Air convection coefficient is $50\left[\mathrm{Wm}-{ }^{2} \mathrm{~K}^{-1}\right]$ 
The origin of an $x-y-z$ coordinate system was considered at the centre of the laser beam on the work piece surface. The depth of the work piece was aligned in $\mathrm{z}$ direction and increases with increasing $z$. The workpiece moves in the $-y$ direction with a constant velocity $U$ as shown in Figure 1 .

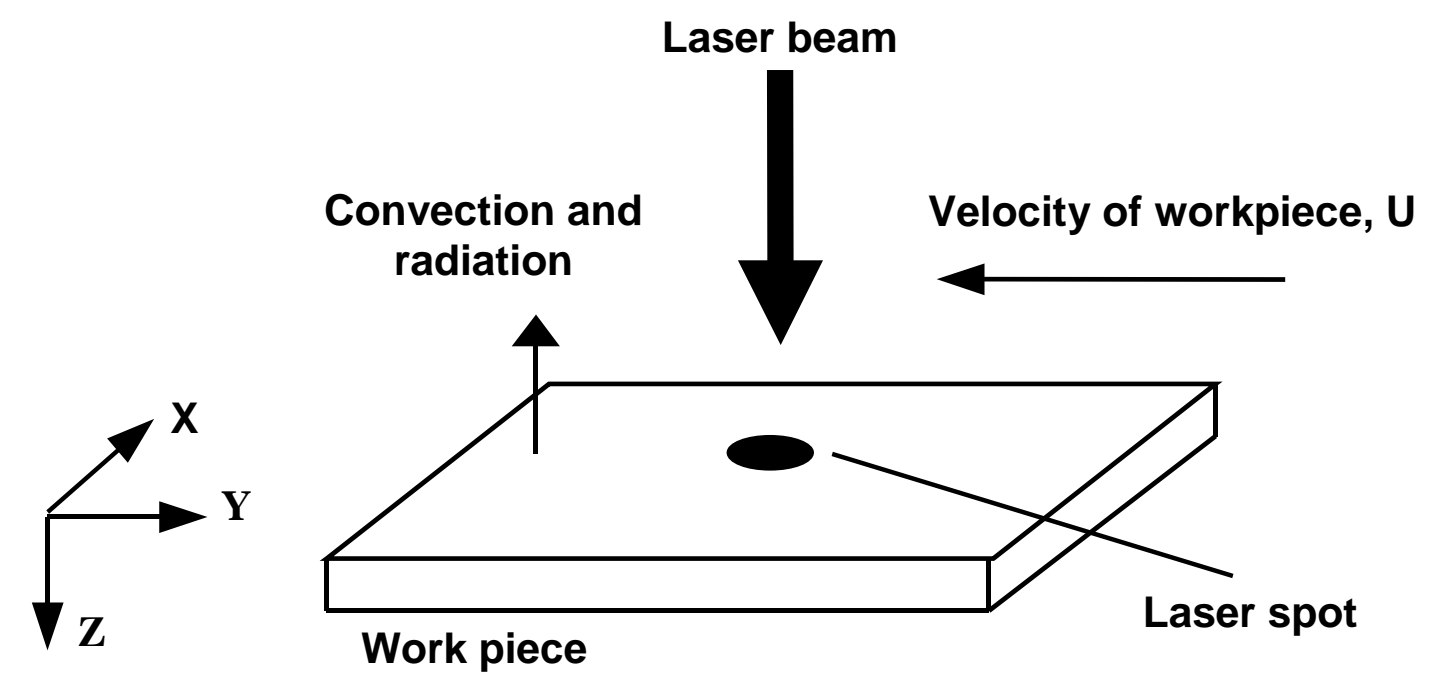

Figure1. Schematic model of the laser heating trial. The laser beam and the coordinate system are fixed and the work piece moves at velocity $U$.

The 3-D transient time-dependent heat conduction in the space underneath the irradiated surface is described by the Equation [9]:

$$
\underbrace{\rho c_{p}\left(\frac{\partial T}{\partial t}-U \frac{\partial T}{\partial y}\right)}_{\text {storage }}=\underbrace{\frac{\partial}{\partial x}\left(k \frac{\partial T}{\partial x}\right)+\frac{\partial}{\partial y}\left[k \frac{\partial T}{\partial y}\right]+\frac{\partial}{\partial z}\left[k \frac{\partial T}{\partial z}\right]}_{\text {conduction }}+\underbrace{\underset{Q}{Q}}_{\text {generation }}
$$

Where $\rho, c_{p}, k, \dot{Q}, U$ are the density, specific heat, thermal conductivity, rate of volumetric heat generation and laser scan speed, respectively.

The initial condition at time $t=0$ is given as

$T(x, y, z, 0)=T_{0}$.

The natural boundary condition takes into account the imposed heat flux, radiation and convection at the laser irradiated surface and is given by

$$
k \frac{\partial T}{\partial n}-q+h\left(T-T_{0}\right)+\sigma \varepsilon\left(T^{4}-T_{0}^{4}\right)=0
$$

Where $h$ is heat transfer coefficient for convection, $\sigma$ is Stefan-Boltzmann constant for radiation $\left(5.67 \times 10^{-8} \mathrm{~W} \mathrm{~m}^{-2} \mathrm{~K}^{-4}\right)$ and $\varepsilon$ is emissivity.

The boundary conditions at the remaining surfaces in the model mimic the actual block. Since only a small portion of the actual block is modelled, the heat flux on the model surfaces is assumed to be approximately equal to the heat conducted from the smaller portion of the block into the bulk.

A thermal numerical simulation was performed to predict the temperature, size and shape of the HAZ. The finite element model was created in ANSYSs (version 11.0 SP1) and is shown in Figure 3. A very finely mapped mesh was used in the area where the Gaussian laser heat flux was incident. This fine mesh method enable the calculation of the steep temperature gradients as accurately as 
possible. The mesh consisted of 8-node 3-D thermal element. Two planes of symmetry were used so that only quarter of the workpiece was modelled. The dimension of the workpiece modelled was $10 \times 20 \times 10 \mathrm{~mm}$. The model contains 184,789 nodes and 124,794 elements. The moving laser heat source is symmetric and hence the quarter-circular Gaussian distribution of heat flux was defined. The initial temperature was $295 \mathrm{~K}$. The bottom face was maintained at $295 \mathrm{~K}$.

\section{Model Results and Validation}

A contour plot of the temperature distribution on the front face of the block is shown in Figure 2 (a) for the case of $678 \mathrm{~W}$ laser power and $17.35 \mathrm{~mm} / \mathrm{sec}$ laser scan speed. The maximum temperature for the case shown was $2333.6^{\circ} \mathrm{C}$ and the temperature at a distance of $0.74 \mathrm{~mm}$ from the centre of the laser beam along the $Z$-axis is about $980^{\circ} \mathrm{C}$. This indicates that the temperature distribution in the quarter circular region decrease very rapidly. Figure 2 shows a close correlation between the simulation (Figure 2 (a)) and experimental results (Figure 2(b)) concerning the prediction of the HAZ. Some slight differences can be seen. The simulation predict a slightly shallow depth $(0.82 \mathrm{~mm}$ for simulation, $0.83 \mathrm{~mm}$ for experimental) and wider width $(3.12 \mathrm{~mm}$ for the simulation, $3.26 \mathrm{~mm}$ for the experimental). Therefore, for a given HAZ volume, the shape of the predicted HAZ is slightly different from the experimentally determined geometry.

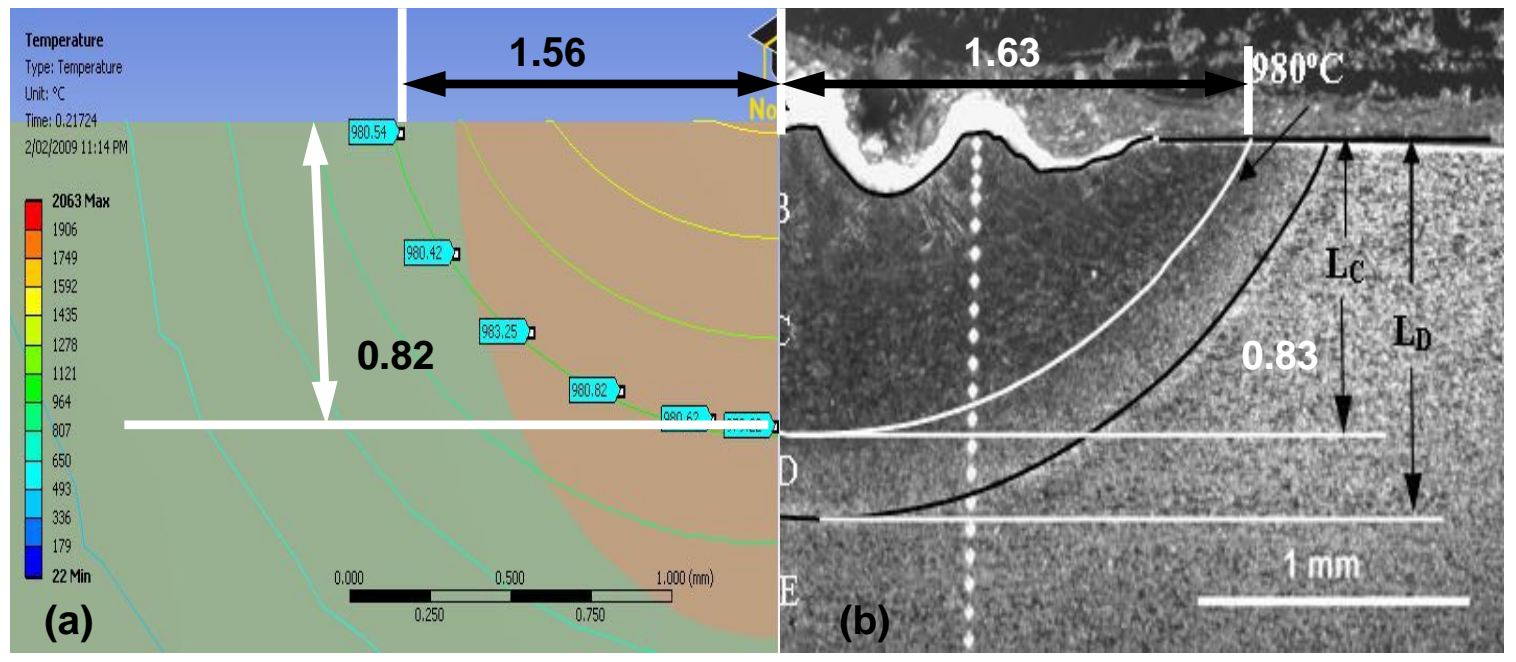

Figure 2. Comparision of the geometry of the simulation and experimental $\mathrm{HAZ}$ for a $678 \mathrm{~W}$ laser power, $17.35 \mathrm{~mm} . \mathrm{sec}^{-1} \mathrm{scan}$ speed, $4.4 \mathrm{~mm}$ spot size.

\section{Conclusions}

- The transient three-dimensional finite element model has been successfully developed to analyze the temperature distribution in the Ti6Al4V alloy workpiece and geometry of the HAZ.

- The results of the models have been compared with the experimental results. A good agreement between theory and experiment has been determined.

- The thermal model accurately predicts the volume of material with a temperature high than $980^{\circ} \mathrm{C}$, which corresponds to the HAZ . The size of the HAZ is a function of the laser power, speed, and spot size.

- The thermal model can be used to determine the laser parameters for a given cut geometry that will yield no residual HAZ in the material after cutting.

- The results reported here using the thermal model are only preliminary results. More work needs to be carried out to ensure that the model fully describes the physical situation. 
- A set of calibration runs would be useful to ascertain the magnitude of systematic errors inherent in the experiment and laser parameters.

\section{Acknowledgements}

The authors would like to thankfully acknowledge support of the CAST CRC, established and supported by the Australian Government's Cooperative Research Centres Programme, and IRIS, Swinburne University of Technology.

\section{References}

[1] Rahman, M. and Wang, Z. G., et al. (2006) A review on high-speed machining of titanium alloys, Jsme International Journal Series C-Mechanical Systems Machine Elements and Manufacturing 49, 11-20.

[2] Chryssolouris, G., Anifantis, N. and Karagiannis, S. (1997) Laser assisted machining: an overview, ASME, Journal of Manufacturing Science and Engineering 119, 766-769.

[3] Lei, S., Shin, Y.C and Incropera, F.P. (1999) Experimental investigation on thermomechanical characteristics in laser-assisted machining of silicon nitride ceramics, Proceedings of ASME, Manufacturing Science and Engineering, MED 10, 781-788.

[4] Anderson, M. and Patwa,R. et al. (2006) Laser-assisted machining of Inconel 718 with an economic analysis, International Journal of Machine Tools \& Manufacture 46, 1879-1891.

[5] Internet Literature:

http://www.industrial-lasers.com/display_article/313470/39/none/none/Feat/Laser-assistedmachining-of-titanium-alloys.

[6] Germain, G., Morel, F. and Lebrun, J. L. et al. (2007) Machinability and Surface Integrity for a Bearing Steel and a Titanium Alloy in Laser Assisted Machining, Laser in Engineering, 17, 329-344.

[7] Sun, S., Durandet, Y. and Brandt, M. (2007) Laser Assisted Machining of Titanium Alloyslaser preheat model and laboratory trials, CAST report (August), CRC, Swinburne University of Technology.

[8] Singh, R., M. J. Alberts, et al. (2008) Characterization and prediction of the heat-affected zone in a laser-assisted mechanical micromachining process, International Journal of Machine Tools \& Manufacture 48, 994-1004.

[9] Incropera, F.P. and Dewitt, D.P. et al (2006) Fundamentals of Heat and Mass Transfer, $6^{\text {th }}$ ed. John Wiley \& Sons, New York, 72-73. 